\title{
Revisit epoch duration for sake of patient-friendly sleep studies
}

\author{
Jaspal Singh $^{1}$ (D) R. K. Sharma ${ }^{2}$ \\ Received: 13 December 2018 / Accepted: 11 March 2019 / Published online: 15 March 2019 \\ (c) Japanese Society of Sleep Research 2019
}

Keywords Epoch duration $\cdot$ Sleep staging $\cdot$ Physiological coupling $\cdot$ Home monitoring

\section{Dear Editor,}

Staging was recommended for each 30-s epoch of the sleep in the R\&K framework. The reason for choosing this duration was primarily the then used recording system that presented $30 \mathrm{~s}$ record on a page. The epoch duration has stood the test of time. Although the present computerized PSG systems can easily display any length of signals on a single page (making the original single-page logic irrelevant), no need has been flagged by the clinicians for its reconsideration.

In contrast, looking from the patient point of view, the sleep studies are quite inconvenient and an unobtrusive/wearable system for sleep studies is highly desirable. Fortunately, recent research and advanced electronics is promising exactly the same $[1,2]$. For a new line of assessment, considerations of epoch duration are normal in the literature [3]. So, a relook for the benefit of new breed of patient-friendly systems is worthwhile.

One of the most promising technologies amiable to wearable instrumentation for sleep-studies is based on automated sleep staging with cardiorespiratory signals. It leverages certain features of the cardiorespiratory signals and their coupling that varies with sleep stages. Complex computations are used to extract the features from signals of $30 \mathrm{~s}$ length and classifiers are then used for staging. The limited length of signals affects the accuracy of computational processes [4]. The use of longer signals should enable better estimation of rhythms and has been successfully used by researchers for sleep staging [5]. Although this research is still a long way from replacing the standard PSG but the promise of automated staging and unobtrusive studies is commanding.

Jaspal Singh

jaspal_sng@yahoo.com

1 Electronics for Medicine and Biology, CDAC, $\mathrm{Ph}-8$, Mohali 160058, India

2 Electronics and communications Engineering, National Institute of Technology, Kurukshetra, Haryana, India
While there may be no clinical need, but what if staging epoch is made $60 \mathrm{~s}$ ? For one, transition marking may get hazy. Again, would it affect clinical outcome? The topic is debatable and worthy of fresh research. In any case, such increase may halve the time spent in manual staging/review. It may help promote the research in cardiorespiratory sleep staging for patient-friendly unobtrusive in-home studies.

Sincerely,

Jaspal Singh \& R K Sharma

Acknowledgements No funds have been received for this work.

\section{Compliance with ethical standards}

Conflict of interest The authors declare no conflict of interests.

\section{References}

1. Uchida S, Endo T, et al. Sleep evaluation by a newly developed PVDF sensor non-contact sheet: a comparison with standard polysomnography and wrist actigraphy. Sleep Biol. Rhythms 2011;9(3):178-187.

2. Fujimoto K, Ding Y, Takahashi E, Sleep stage detection using a wristwatch-type physiological sensing device. Sleep Biol. Rhythms, 2018;16(4):449-456.

3. YAN M-M, XU X-H, et al. Selection of optimal epoch duration in assessment of rodent sleep-wake profiles. Sleep Biol. Rhythms 2011;9(1):46-55.

4. Pinna GD, Maestri R, Sanarico M. Effects of record length selection on the accuracy of spectral estimates of heart rate variability: a simulation study. IEEE Trans Biomed Eng. 1996;43(7):754-7.

5. Willemen T, et al. An evaluation of cardiorespiratory and movement features with respect to sleep-stage classification. IEEE J Biomed Heal Inform. 2014;18(2):661-9.

Publisher's Note Springer Nature remains neutral with regard to jurisdictional claims in published maps and institutional affiliations. 